\title{
Terminologia de referência em Enfermagem: a Norma ISO 18104
}

\author{
Reference terminology model for nursing: the ISO 18104 standard \\ Terminologia de referencia en Enfermería: la Norma ISO 18104
}

\section{Heimar de Fátima Marin ${ }^{1}$}

\section{RESUMO}

O presente artigo descreve a Norma ISO 18104, publicada em 2003, pelo comitê técnico TC 215 da ISO. Esta norma contempla o modelo de terminologia de referência para diagnóstico e ações de enfermagem. A terminologia de referência representa um instrumento para facilitar o mapeamento entre as diversas terminologias existentes, facilitando a integração dos sistemas de informação e prontuários eletrônicos. Descritores: Terminologia; Sistemas computadorizados de registros médicos/normas; Diagnóstico de enfermagem/normas; Informática em enfermagem

\section{ABSTRACT}

This article describes the ISO 18104 standard, published in 2003 by the International Organization for Standardization Technical Committee 215 (ISO/TC). The standard consists of reference terminology model for nursing diagnoses and interventions. The reference terminology is an instrument that facilitates the mapping of several available terminologies, which promotes the integration of the healthcare information systems and electronic medical records.

Keywords: Terminology; Medical records systems, computerized/standards; Nursing diagnosis/standards; Nursing Informatics

\section{RESUMEN}

El presente artículo describe la Norma ISO 18104, publicada en el 2003, por el comité técnico TC 215 de la ISO. Esta norma contempla el modelo de terminología de referencia para el diagnóstico y acciones de enfermería. La terminología de referencia representa un instrumento para facilitar la identificación entre las diversas terminologías existentes, facilitando la integración de los sistemas de información e historias clínicas electrónicas.

Descriptores: Terminologia; Sistemas de historias clínicas informatizadas/normas; Diagnóstico de enfermería/normas; Informática y enfermería

\footnotetext{
${ }^{1}$ Professora Titular da Universidade Federal de São Paulo - UNIFESP - São Paulo (SP), Brasil.
} 


\section{INTRODUÇÃO}

O prontuário do paciente representa o principal veículo de comunicação formal entre os membros da equipe de saúde. É através deste registro que enfermeiros, médicos e demais profissionais envolvidos com o atendimento a um paciente podem compartilhar a informação e assegurar a continuidade do cuidado. É preciso que a informação seja legível, objetiva, clara e completa, de forma que todos os membros da equipe de saúde entendam o contexto e o significado da mesma. Embora no Brasil, a maior parte dos prontuários siga o modelo de documentação manual e não eletrônica, alguns centros já previlegiam o registro eletrônico e o uso de padrões de registro para a documentação clínica.

O uso de padrões para o registro eletrônico é fundamental para a recuperação e análise da informação, mas ainda representa um grande desafio, principalmente para os profissionais que realizam a documentação na beira do leito, considerando que um vocabulário que padronize os termos clínicos para uso na prática diária deve atender critérios como validade, especificidade, recuperação dos dados e facilidade de comunicação. Acrescenta-se ainda que, um vocabulário clínico deve ser simples de entender, fácil de codificar e intuitivo aos profissionais de saúde.

$\mathrm{Na}$ enfermagem, cerca de treze vocabulários foram desenvolvidos, sendo que no Brasil, os mais conhecidos e utilizados são: os diagnósticos da NANDA (Associação Norte Americana de Diagnósticos de Enfermagem North American Nursing Diagnoses Association), a NIC (Classificação das Intervenções de Enfermagem), a NOC (Classificação de Resultados de Enfermagem) e a CIPE Versão 1.0 (Classificação Internacional para a Prática de Enfermagem).

Ressalta-se, que todos os vocabulários que foram ou estão sendo desenvolvidos possibilitam a documentação de enfermagem de acordo com as etapas do processo de enfermagem. A prática de enfermagem encontra nomes para compor todos estes elementos. A ideia é que estes nomes tenham o mesmo significado para profissionais de diferentes regiões. Uma vez conseguida esta padronização entre os enfermeiros, estas palavras se tornam Nomenclatura de Enfermagem que podem ser combinadas para formar, então, o sistema de linguagem ${ }^{(1)}$. Porém, a utilização de um vocabulário padronizado não significa que a interoperabilidade e a troca de informações entre sistemas computacionais possam ser obtidas. Era comum aos pesquisadores brasileiros e internacionais, a realização do mapeamento entre as diversas terminologias existentes. $\mathrm{Na}$ tentativa de facilitar este processo, foi criada a norma ISO 18104 e este artigo pretende apresentar esta norma para que futuros estudos de aplicabilidade possam ser feitos, analisando potenciais usos da referida norma nos sistemas já desenvolvidos pelos pesquisadores do país.

\section{A NORMA ISO 18104}

A ISO (International Organization for Standard Organização Internacional de Padronização) é uma federação mundial de normalização que conta com a participação de representantes de diversos países, composta de diversos comitês que têm o trabalho de preparar normas internacionais. A ISO 18104 foi preparada pelo Comitê Técnico ISO/TC215(2) de Informática em Saúde, por meio de uma proposta de item de trabalho, iniciada pelo Grupo de Interesse Especial de Informática em Enfermagem da Associação de Informática Médica Internacional (IMIANISIG www.imiani.org) e pelo Conselho Internacional de Enfermagem.

O propósito da ISO 18104 - Modelo de Terminologia de Referência ${ }^{(2)}$ para a Enfermagem é acomodar as várias terminologias e classificações atualmente usadas pelos enfermeiros para a documentação de dados dos pacientes e clientes. Uma terminologia de referência com representação de conceitos facilita o mapeamento dos termos de enfermagem com outras terminologias de saúde, promovendo a integração dos sistemas de informação. Desde o início, a criação das terminologias de enfermagem foi motivada pela necessidade de obter um formato de registro compatível com a necessidade de processamento computacional, viabilizando pesquisas comparativas e análises de resultados a fim de melhorar e fortalecer o corpo de conhecimento da enfermagem.

Um modelo de terminologia de referência pode servir de instrumento para facilitar a representação dos diagnósticos e ações de enfermagem e seus relacionamentos, de forma que se possa realizar análise computacional, promovendo avaliações sistemáticas das terminologias e dos modelos existentes, facilitando a harmonização entre as diversas terminologias em uso, para fornecer uma linguagem que descreva a estrutura dos conceitos dos diagnósticos e ações de enfermagem, de forma a integrar os modelos de informação ${ }^{(3)}$. As terminologias de enfermagem, tanto no formato baseado em papel quanto eletrônico, foram projetadas como classificações enumeradas e implantadas como terminologia de interface no ponto do cuidado e como terminologia administrativa para examinar dados de enfermagem nos diversos setores. Até hoje, apesar da variedade de terminologias desenvolvidas, nenhuma delas é completa o suficiente em termos de abrangência e granularidade. Além disso, não existe uma terminologia orientada pelo conceito que integre o domínio dos conceitos de enfermagem de uma forma adequada para 
o processamento eletrônico ${ }^{(2)}$

Modelo de terminologia de referência para o diagnóstico de enfermagem

$\mathrm{Na}$ norma ISO 18104, um diagnóstico de enfermagem é considerado como um julgamento em um foco ou como um julgamento em uma dimensão particular de um foco (por exemplo, habilidade, conhecimento). Uma representação gráfica de um modelo de terminologia de referência para diagnóstico de enfermagem é mostrado na Figura 1. Um descritor para foco e um descritor para julgamento são mandatórios para a definição de um diagnóstico de enfermagem. Em algumas instâncias especiais, um descritor único (por exemplo, dor) pode servir para o papel de ambos, foco e julgamento. Nenhuma categoria básica é especificada; a decisão está a cargo do desenvolvedor de terminologia e/ou do profissional que está desenvolvendo um sistema de informação e implantando uma terminologia.

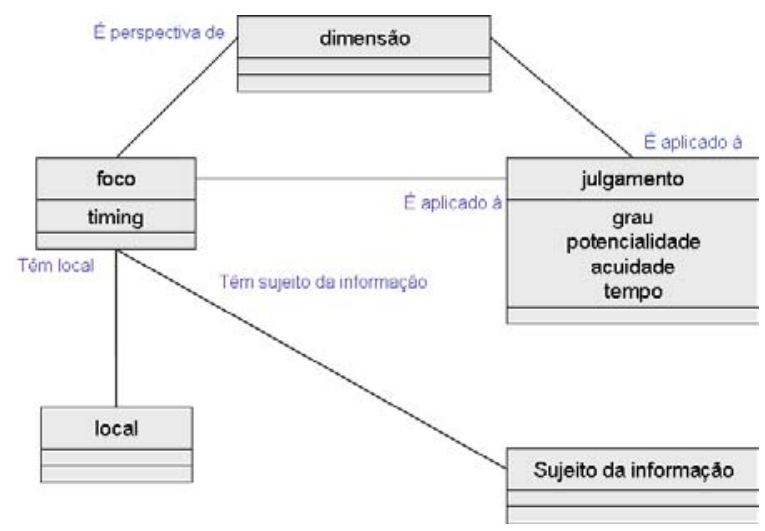

Figura 1 - Modelo de terminologia de referência para diagnóstico de enfermagem

Para melhor ilustrar o modelo acima, segue-se um exemplo:

Não aderência ao regime medicamentoso

Dimensão: regime medicamentoso

Tem julgamento: inadequado

Tem qualificador de potencialidade: atual

Tem sujeito da informação: cliente

Modelo de terminologia de referência para ação de enfermagem

Uma ação é um processo pelo qual um serviço intencional é aplicado a um recebedor de cuidados. Ações são freqüentemente representadas em expressões compostas de verbos ou expressões verbais. Ação pode ser qualificada pelo tempo. Exemplos de descritores incluem observação, ensino, prevenção e alimentação. A Figura 2 apresenta o modelo de terminologia de referência para ações de enfermagem

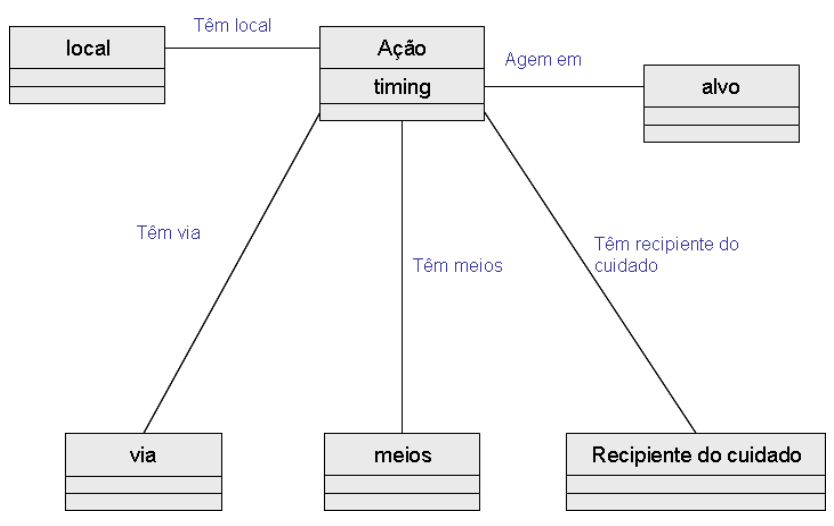

Figura 2 - Modelo de terminologia de referência de enfermagem para ações de enfermagem

Novamente, com o intuito de ilustrar o modelo acima, segue-se um exemplo:

Aspirar secreções de vias aéreas pela traquéia

Ação: Aspirar

Age em: secreções

Tem local: vias aéreas

Tem via: pela traquéia

Tem recipiente de cuidado: cliente

\section{COMENTÁRIOS FINAIS}

Embora o interesse no desenvolvimento de padrões no vocabulário seja grande, alguns autores identificam algumas barreiras na concretização de seus objetivos. Estas barreiras são apresentadas na forma de múltiplos marcos conceituais que regem a enfermagem, na identificação dos elementos do arquivo mínimo de enfermagem, nos sistemas de classificação para diagnóstico, intervenção e resultados, na própria falta de um código uniforme e até mesmo na perspectiva da visão global do indivíduo feita pelos enfermeiros. Com a criação de múltiplos vocabulários em enfermagem, tornou-se necessário desenvolver um Modelo de Referência a ser considerado como uma base comum para o registro, transferência e análise dos dados de enfermagem.

Hoje, a informação é considerada o elemento essencial para ação com qualidade. Quem mais acesso possui à informação, melhor qualidade de trabalho consegue produzir. A informação, via de regra, nos chega na forma eletrônica e os sistemas computacionais estão, cada vez mais desenvolvidos para sustentar o registro e análise das informações em saúde. Os países estão se organizando na construção das bases de dados nacionais, onde toda a informação clínica de um cidadão está cadastrada e pode ser compartilhada entre os membros da equipe de saúde. Tais bases de dados permitirão análise e avaliação de procedimentos, tratamentos e cuidados, de modo a identificar a melhor prática. Precisamos, portanto, garantir que os dados e as informações que apoiam e descrevem 
a prática de enfermagem estejam presentes nestas bases de dados e que, de fato, tais dados representem a real

\section{REFERÊNCIAS}

1. International Council of Nurses. Nursing's next advance: an international classification for nursing practice (ICNP) - a working paper. Genebra: ICN; 1993.

2. International Organization for Standardization - ISO 18104. Health Informatics integration of a reference atividade de enfermagem a serviço de melhores condições de vida e saúde para a população.

terminology model for nursing, Genebra, ISO; 2003. p.128.

3. Saba VK, Hovenga E, Coenen A, McCormick K, Bakken S. Nursing language - terminology models for nurses. ISO Bull. 2003; (September):16- 8. 